\title{
Perfil de internações psiquiátricas femininas: uma análise crítica
}

Profile of female hospitalizations in a psychiatric unit: a critical analysis

DOI: 10.1590/0047-2085000000275

J Bras Psiquiatr. 2020;69(3):165-70

Where you read:

Received in: Apr/28/2020. Approved in: May/11/2020

Should read:

Received in: Apr/19/2020. Approved in: May/11/2020

DOI: $10.1590 / 0047-2085000000297$ 